\title{
Long-term follow-up of posterior capsule opacification after AquaLase and NeoSoniX phacoemulsification
}

\author{
Alexandr Stepanov, Jana Nekolova, Nada Jiraskova, Pavel Rozsival
}

\begin{abstract}
Aim. To compare the degree of posterior capsule opacification (PCO) after AquaLase and NeoSoniX phacoemulsification methods during an 8-year follow-up period using two types of software.

Design. Prospective, randomized clinical trial.

Methods. AquaLase was used in the right eye and NeoSoniX in the left eye of each patient with bilateral cataract.

Results. Fifty patients were analyzed 1 year, 46 patients 3 years, and 37 patients 8 years after cataract surgery. Mean EPCO 2000 values were for the AquaLase group $0.324 \pm 0.305$ and for the NeoSoniX group $0.298 \pm 0.341(P=0.53)$ 1 year after surgery, for the AquaLase group $0.582 \pm 0.506$ and for the NeoSoniX group $0.594 \pm 0.515$ (P=0.87) 3 years after surgery, and for the AquaLase group $0.648 \pm 0.567$ and for the NeoSoniX group $0.673 \pm 0.542(P=0.30) 8$ years after surgery. The OSCA results were for the AquaLase group $0.7097 \pm 0.3778$ and for the NeoSoniX group $0.8584 \pm$ $0.4323(P=0.046) 1$ year after surgery, for the AquaLase group $0.9667 \pm 0.736$ and for the NeoSoniX group $0.9540 \pm$ $0.5250(P=0.91) 3$ years after surgery, and for the AquaLase group $1,035 \pm 0,952$ and for the NeoSoniX group $1,103 \pm$ $0,741(P=0.44) 8$ years after surgery.
\end{abstract}

Conclusion. There was minimal PCO difference between these 2 approaches, AquaLase and NeoSoniX. Neither AquaLase nor NeoSoniX technique was able to prevent a natural progression of PCO.

Key words: posterior capsule opacification, AquaLase, NeoSoniX

Received: April 9, 2015; Accepted: July 23, 2015; Available online: September 3, 2015

http://dx.doi.org/10.5507/bp.2015.039

Department of Ophthalmology, Faculty of Medicine in Hradec Kralove, Charles University in Prague and University Hospital Hradec Kralove, Czech Republic

Corresponding author: Alexandr Stepanov, e-mail: stepanov.doctor@gmail.com

\section{INTRODUCTION}

Posterior capsule opacification (PCO) is one of the most common complications of cataract extraction with intraocular lens (IOL) implantation, occurring in 36\% to $97 \%$ of cases in the first $2-4$ years after extracapsular cataract surgery ${ }^{1-6}$. Since the PCO usually develops over years and leads to a slow decrease in visual acuity (VA), progression over time is the important factor in PCO evaluation ${ }^{7}$. Advances in surgical technique and IOL design may decrease the amount of PCO after cataract extraction. In recent years, perioperative pharmacological modifications and changes in IOL design have been made to reduce the incidence of PCO (ref. ${ }^{8,9}$ ).

However, the most critical factors to reduce PCO involve removal of as many residual epithelial cells as possible, proper placement of the IOL inside the capsular bag, a capsulorrhexis size that is smaller than the IOL optics, and use of an IOL with sharp-edged optics ${ }^{10,11}$.

The goal of this study was to examine the progression of PCO through 8 years in eyes after 2 different irrigation/aspiration techniques (AquaLase and NeoSoniX) of cataract surgery in which AcrySof SA60AT (AlconLaboratories, Fort Worth, Texas, USA) sharpedged silicone intraocular lenses. We used the Evaluation of Posterior Capsule Opacification (EPCO) 2000 software (Berlin, Germany) for morphologic scoring of PCO and the Open-Access Systematic Capsule Assessment (OSCA) system (Edinburgh, United Kingdom) for PCO analysis.

The previous results of this study after 1, 2 and 3-year follow-up have been published ${ }^{12}$. In order to investigate whether the difference in PCO rates after AquaLase and NeoSoniX phacoemulsification was consistent over longer period of time, an 8-year follow-up was performed.

\section{METHODS}

Study design, image acquisition, and computer analysis of PCO, as well as the statistical methods and sample size calculation used, were exactly the same as in our previous publication ${ }^{12}$. Thirty-seven patients of the original 62 were included in the study. The inclusion criteria were bilateral nonbrunescent cataract (according to the Buratto classification, a cataract grade of less than 5) (ref. ${ }^{13}$ ) and no other severe ocular pathologic features potentially affecting VA (patients with mild age-related macular degeneration were not excluded). Both eyes of each patient involved in the study had cataract with similar density grade. All surgeries were performed in the years 2004 and 2005 at the Department of Ophthalmology, University Hospital in Hradec Kralove. The AquaLase was used in the right eye and NeoSoniX was used in the left eye of 
each patient ${ }^{12,14}$. All surgeries were performed by 1 of 2 skilled surgeons (N.J., P.R.); both eyes of 1 patient were operated on by the same surgeon. The same IOL, AcrySof SA60AT (Alcon Laboratories, Fort Worth, Texas, USA), was implanted in all eyes. All participants were asked to come for examination 1, 3 and 8 years after surgery. In all patients able to participate in the follow-up, a complete eye examination was performed and digital retroillumination photographs, after attaining maximal pupil dilation, were obtained using the SL 990 slit-lamp equipped with Digital Vision System (CSO, Florence, Italy). The PCO quantification was done using the subjective Evaluation of Posterior Capsule Opacification (EPCO) 2000 software (Developed by Tetz MR and associates, Berlin, Germany) and the objective Open-Access Systematic Capsule Assessment (OSCA) system of computer PCO analysis (Devised by Aslam TM, Edinburgh, United Kingdom). Both systems enabled the further comparison of PCO after AquaLase and NeoSoniX cataract removal techniques. Eyes with preceding $\mathrm{Nd}$ :YAG laser capsulotomy were excluded from the computer PCO assessment. The remaining images were computer analyzed. They were imported into the EPCO 2000 program and the PCO was evaluated for the entire optic. The opacification density was graded as minimal (grade 1) when the capsule showed mild wrinkling, mild homogenous layers, or sheets of lens epithelial cells. Areas of honeycomb patterns of PCO, thicker homogenous layers, and denser fibrosis were graded as mild (grade 2), whereas areas of classical Elschnigg pearls and of very thick homogenous layers were graded as moderate (grade 3 ). Areas of very thick Elschnigg pearls with "darkening effect" or of any type of severe opacification were graded as severe (grade 4). The individual PCO score was calculated by multiplying the opacification grade by the fraction of capsule area involved behind the IOLs optic ${ }^{15}$. The images then were analyzed with the OSCA system. It is possible to use 3 different methods of analysis: 1) the Single Analysis, for analyzing the only image of the patient's capsule with flash not covering the PCO; 2) the New Analysis, requiring 2 images containing spoiled flash areas in different regions; and 3 ) the Circ Analysis, which allows the specification of the central number of pixels to be measured ${ }^{16}$. In this study, the Single Analysis was used when the flash light did not cover PCO and the New Analysis was used when the flash had to be removed without losing potential PCO areas under the flash. The EPCO indexes and the OSCA scores were calculated automatically and statistically analyzed. Best-corrected visual acuity (BCVA) was measured using Snellen optotypes before and 1, 3, and 8 years after surgery. Nd:YAG laser capsulotomy incidence was evaluated. Nd:YAG capsulotomy was performed based on subjective patient disappointment regarding the quality of his or her vision and measurable decrease in VA compared to best postoperative VA (loss of more than 1 line in Snellen BCVA), coupled with the presence of PCO in the central part of posterior capsule. Measurement of the degree of capsule opacification was not determinant. Statistical analysis was performed using MS Excel (Microsoft Corp, Redmond, Washington, USA) and NCSS (the Statistical and Power Analysis software) (NCSS, Kaysville, Utah, USA). Paired t test was used to compare EPCO indexes and OSCA scores, each for the right eye and the left eye 1,3 , and 8 years after surgery. Simple linear regression was performed for the assessment of the relations of total EPCO index to postoperative BCVA and OSCA scores to postoperative BCVA. Correlation between EPCO and OSCA results was assessed using Pearson correlation coefficient test. All decisions were made at significance level (alpha) of 0.05 .

\section{RESULTS}

Sixty-two patients eligible for the study were originally included. Fifty patients completed 1-year follow-up examination, 46 of them attended 1-year and 3-year follow-up examinations, and 37 participants completed all 8-year follow-up examinations. Nd:YAG laser capsulotomy rate for AquaLase vs NeoSoniX was 0:1 eyes 1 year postoperatively, 1:4 eyes 3 years after surgery and 2:6 eyes after 8 years. Thus, the PCO of 49 patients were computer analyzed 1 year after surgery, 42 patients were included in the computer PCO analysis 3 years postoperatively, and PCO of 37 patients were computer analyzed after 8-year follow-up.

The mean OSCA scores, the mean total EPCO 2000 indexes, and $P$ values of paired t test analysis, each for the right eye operated on by using AquaLase phacoemulsification and for the left eye after NeoSoniX cataract removal method, are shown in Table 1. There are 1-year, 3-year, and 8-year-results.

The summary graph illustrating development of PCO after the AquaLase and the NeoSoniX method for each measurement type, EPCO 2000 and OSCA, within the 8 -year follow-up period is shown in Fig. 1. The comparison of PCO after AquaLase and NeoSoniX phacoemulsification is shown in Fig. 2. There are mean total EPCO 2000 indexes and OSCA scores, with error bars.

An increase in EPCO 2000 indexes was found during the 8-year period, after both AquaLase and NeoSoniX cataract removal method $(P<0.05)$. A significant increase was proved in the EPCO 2000 outcomes between the first and third postoperative years. The difference in the EPCO 2000 scores between the third and eight postoperative years was not significant.

The difference in the OSCA indexes between the first and third and between the third and eight postoperative years was significant; the scores was nonsignificantly higher in the AquaLase group, 8 years after surgery $(P=$ $0.41)$, whereas in the NeoSonix group it was significantly higher in the eight year $(P=0.68)$. A significant increase was proved in the OSCA outcomes between the first and the third postoperative year, after both AquaLase and NeoSoniX phacoemulsification method $(P<0.05)$.

The comparison of PCO after the AquaLase and the NeoSoniX technique was done. The EPCO 2000 results were as follows: after 1 year, there was no proven significant difference between the AquaLase and the NeoSoniX group; the mean total EPCO index was slightly better for 
Table 1. Mean Values With Standard Deviation of Total Evaluation of Posterior Capsule Opacification 2000 Indexes and OpenAccess Systematic Capsule Assessment Scores for the AquaLase and the NeoSoniX Group, 1, 3 and 8 Years After Cataract Surgery.

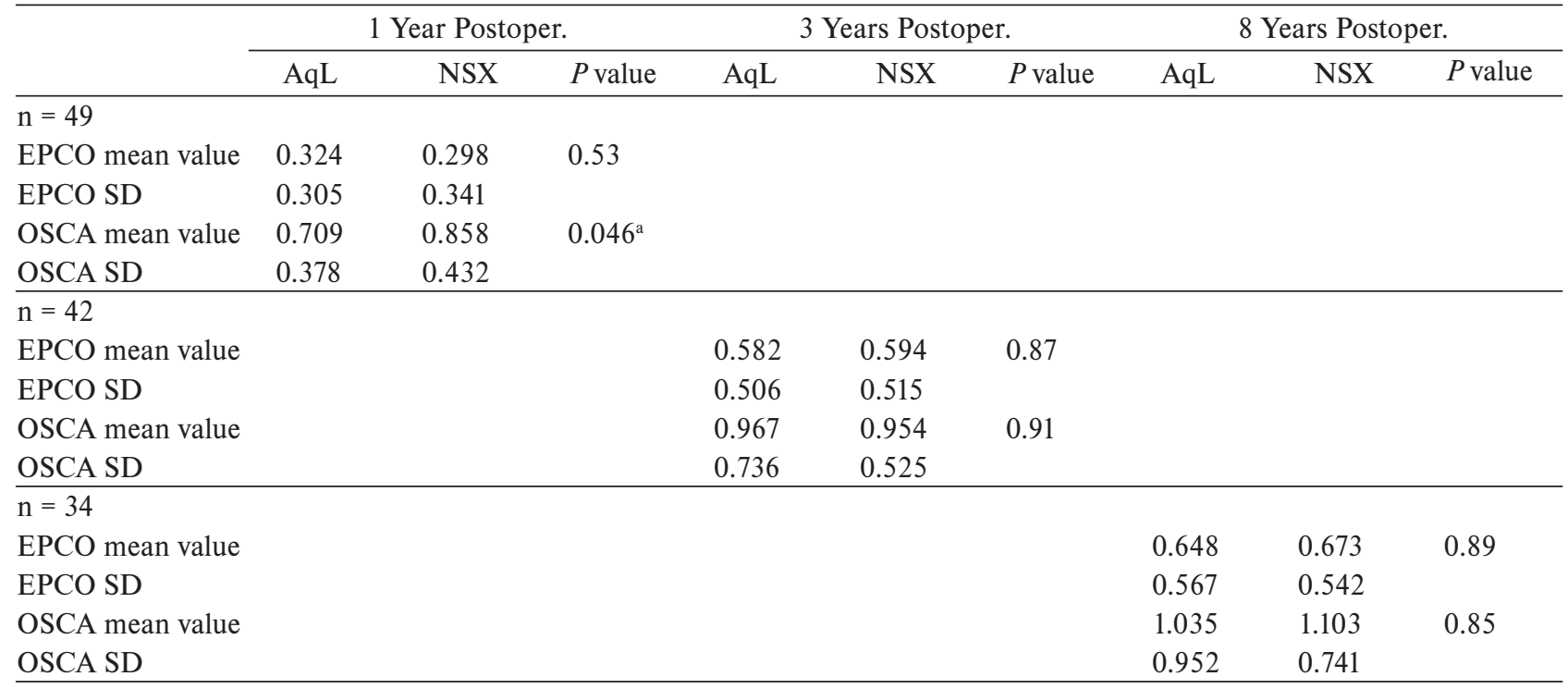

$\mathrm{AqL}=$ AquaLase; $\mathrm{EPCO}=$ Evaluation of posterior capsule opacification; NSX = NeoSoniX; OSCA = Open-Access Systematic Capsule Assessment; $\mathrm{SD}=$ standard deviation.

Comparison of posterior capsule opacification after AquaLase and NeoSoniX; $P$ values of paired $t$ test.

${ }^{a} P<0.05$ (confidential interval alpha) showed a significant difference.

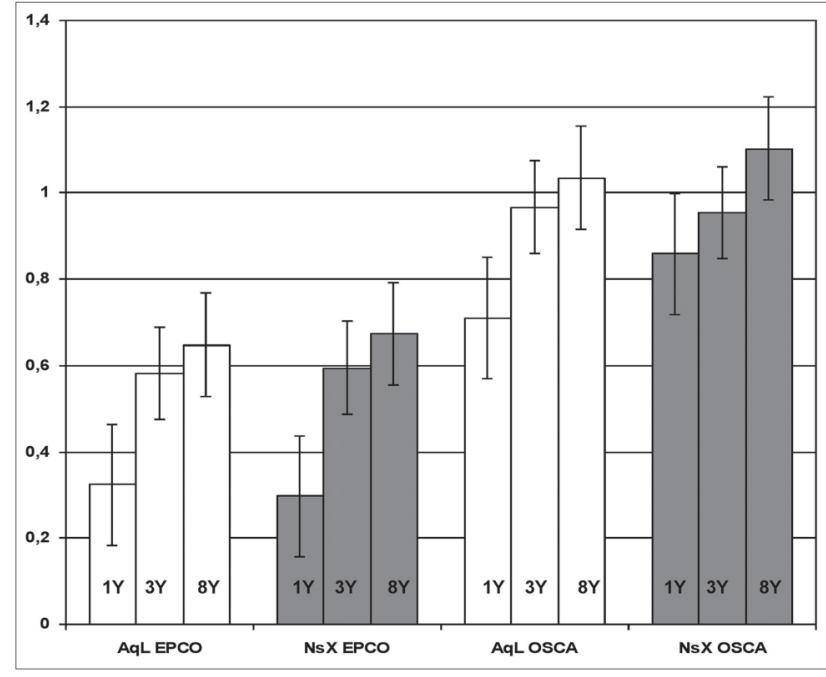

Fig. 1. Summary graph illustrating the development of posterior capsule opacification (PCO) after the AquaLase (AqL) and the NeoSoniX (NSX) method for each measurement type, Evaluation of Posterior Capsule Opacification (EPCO) 2000 and Open-Access Systematic Capsule Assessment (OSCA). Mean values with error bars. One-year (1Y), 2-year (2Y), and 8 -year (8Y) results. Considerable increase in the mean total EPCO indexes was found during the $3 \mathrm{Y}$ period, after both AquaLase and NeoSoniX cataract removal method. The OSCA score was higher in the AquaLase group 3 years after surgery, whereas in the NeoSoniX group it was only nonsignificantly higher in the third year. A nosignificant increase was proved in the EPCO outcomes between the third and the eight postoperative year and in the OSCA outcomes after AquaLase method. The OSCA score was significantly higher in the NeoSoniX group 8 years after surgery. White bars = AquaLase group; gray bars $=$ NeoSoniX group.

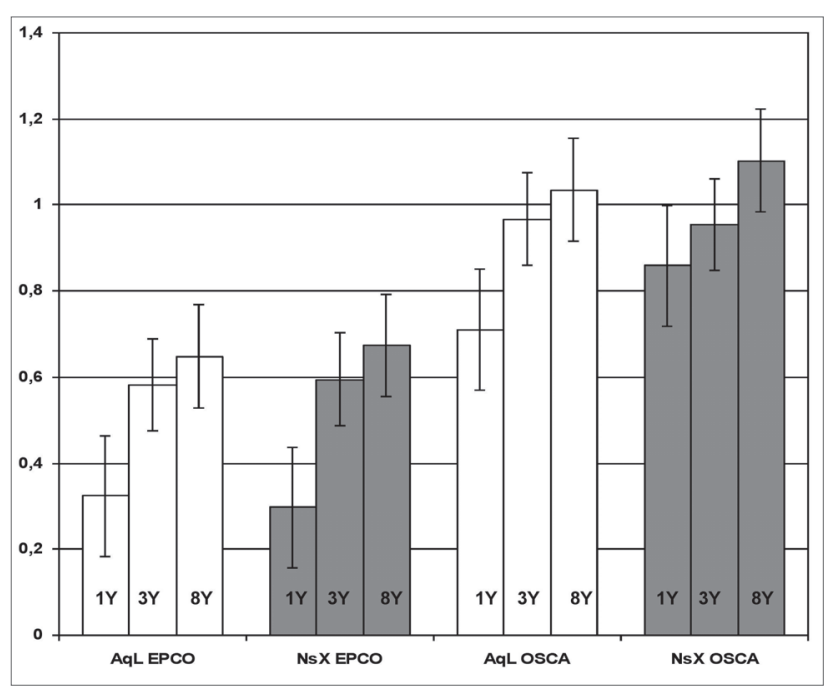

Fig. 2. Comparison of mean total EPCO 2000 indexes and OSCA scores after AqL and NSX phacoemulsification 1, 3, and 8 years after cataract surgery, with error bars. The difference in EPCO 2000 outcomes was not significant. There was a significant difference in OSCA scores 1 year and 8 years postoperatively and nonsignificant difference 3 years postoperatively. White bars $=$ AquaLase group; gray bars $=$ NeoSoniX group . 
Table 2. Mean Evaluation of Posterior Capsule Opacification 2000 Indexes With Standard Deviation of All Grades and of Total Evaluation of Posterior Capsule Opacification Index for AquaLase and NeoSoniX Group, 1, 3, and 8 Years After Cataract Surgery.

\begin{tabular}{|c|c|c|c|c|c|}
\hline & Minimal PCO & Mild PCO & Moderate PCO & Severe PCO & Total EPCO Index \\
\hline \multicolumn{6}{|c|}{ AquaLase } \\
\hline 1 year postoperatively & $0.259 \pm 0.263$ & $0.031 \pm 0.091$ & $0.001 \pm 0.009$ & 0 & $0.324 \pm 0.305$ \\
\hline 3 years postoperatively & $0.255 \pm 0.260$ & $0.085 \pm 0.130$ & $0.035 \pm 0.101$ & $0.012 \pm 0.042$ & $0.582 \pm 0.506$ \\
\hline 8 years postoperatively & $0.310 \pm 0.229$ & $0.108 \pm 0.14$ & $0.047 \pm 0.18$ & 0 & $0.648 \pm 0.567$ \\
\hline \multicolumn{6}{|c|}{ NeoSoniX } \\
\hline 1 year postoperatively & $0.226 \pm 0.296$ & $0.018 \pm 0.059$ & 0 & $0.004 \pm 0.019$ & $0.298 \pm 0.341$ \\
\hline 3 years postoperatively & $0.346 \pm 0.306$ & $0.039 \pm 0.070$ & $0.062 \pm 0.216$ & $0.013 \pm 0.037$ & $0.594 \pm 0.515$ \\
\hline 8 years postoperatively & $0.405 \pm 0.238$ & $0.09 \pm 0.127$ & $0.025 \pm 0.089$ & $0.128 \pm 0.167$ & $0.673 \pm 0.542$ \\
\hline
\end{tabular}

EPCO = Evaluation of Posterior Capsule Opacification; OSCA = Open-Access Systematic Capsule Assessment; PCO = posterior capsule opacification.

the NeoSoniX group. Whereas in 3-year and 8-year results the AquaLase showed better outcomes, the difference was not significant. The mean EPCO 2000 indexes with standard deviation of all EPCO grades and of total EPCO index for the AquaLase and the NeoSoniX group, 1, 3, and 8 years after cataract surgery are shown in Table 2 . There was an increase in PCO density within the whole follow-up period; there were more PCO assessed with higher EPCO grades. The PCO scores obtained with the OSCA system were worse for the NeoSoniX group 1 and 8 years after cataract surgery, unlike the 3-year results, when the mean OSCA score was nonsignificantly better for the NeoSoniX group. There was low correlation between the EPCO and OSCA results, 1,3 , and 8 years after cataract surgery $(P$ value was 0.0046 and correlation coefficient was 0.347 1 year after surgery, 3 years postoperatively $P$ value was 0.00000007 and correlation coefficient was 0.547 , and 8 years after surgery $P$ value was 0.00058 and correlation coefficient was 0.441 ). There was no significant difference found in the BCVA between the AquaLase and the NeoSoniX group. One year after surgery, the BCVA was $0.837 \pm 0.262$ for the right eyes (AquaLase group) and $0.849 \pm 0.224$ for the left eyes (NeoSoniX group). The mean BCVA was $0.886 \pm 0.197$ for the AquaLase group and $0.842 \pm 0.211$ for the NeoSoniX group 3 years after cataract surgery and $0.834 \pm 0.07$ for the right eyes and $0.825 \pm 0.08$ for the left eyes 8 years postoperatively. Most eyes ( $85 \%$ on average) achieved BCVA 0.8 and better $(0.8$ to 1.2$)$.

There was a nonsignificant increase in BCVA between the first and the third postoperative year in the AquaLase group, $P=0.006$; and nonsignificant decrease in the NeoSoniX group, $P=0.11$ ). And between the third and eight year, the BCVA nonsignificant decreased.

\section{DISCUSSION}

Posterior capsular opacification is the most frequent complication of cataract surgery. Advances in surgical techniques, intraocular lens materials, and designs have reduced the PCO rate, but it is still a significant problem ${ }^{17-19}$. Patients with mild age-related macular degeneration were not excluded from this study; this disease might also have an impact on the postoperative visual outcomes.

In our study, the PCO development after 2 different methods of cataract extraction - AquaLase and NeoSoniX - was observed. This is up to knowledge longest period after cataract extraction when the PCO were evaluated by EPCO 2000 and OSCA methods. AquaLase and NeoSoniX are 2 of 4 options provided by INFINITI Vision System (Alcon Laboratories). The major difference is that the AquaLase device does not use ultrasound to break up the lens. Instead, it relies on a relatively highpressure stream of warm (about $60{ }^{\circ} \mathrm{C}$ ), balanced saline solution (BSS) to fragment the nucleus. Several studies have shown that the technology may have applications in polishing the capsule through mechanical washing of epithelial cells from the capsule bag with the fluid pulses ${ }^{20-22}$. The reduction of lens epithelial cells has been shown to help prevent PCO (ref. ${ }^{16}$ ), so AquaLase may carry a reduced risk over standard phacoemulsification cataract extraction for the development of PCO (ref. ${ }^{20,21,23-25}$ ). The AquaLase was primary used for liquefaction of the cataract in this study. We did not use special settings for lens epithelial cells removal, but because it is very difficult to rupture the posterior capsule using the soft AquaLase needle we believe that the polishing of posterior capsule was more accurate in the AquaLase eyes. NeoSoniX is an option that includes a dedicated hand-piece that produces rotary oscillations of the phacoemulsification tip of up to 2 degrees. This oscillatory motion can be used alone or as an adjunct to ultrasonic energy ${ }^{22,26}$. Not many studies have examined the NeoSoniX effect on the PCO alone.

Multiple studies documented that the PCO develops over years. To properly judge the potential of a new IOLs or a surgical method to prevent PCO, a long-term followup (usually 3 years or longer) of the included eyes is necessary $^{27}$. We observed the PCO at 1-year intervals up to 8 years after cataract surgery; therefore the $\mathrm{PCO}$ change over longer period of time was assessed. PCO-induced decrease of VA can be treated by opening the posterior 
lens capsule by a Nd:YAG capsulotomy ${ }^{28}$. After the capsulotomy, computer PCO assessment is no longer possible and these dropouts can considerably bias study results ${ }^{29,30}$. Buehl and associates ${ }^{7}$ published an article in which they identified the difficult statistical problems caused by missing data attributable to Nd:YAG laser capsulotomies in long-term PCO trials, especially when a study includes many capsulotomies. They gave possible statistical solutions to the problem on the basis of data obtained from a representative clinical long-term trial of PCO. In our study, only 6 patients required Nd:YAG capsulotomy over the whole follow-up period. The difference in the laser treatment of PCO was not significant between the AquaLase and the NeoSoniX group, so no direct comparison of software outcomes was made. The capsulotomy dropouts were taken into account when making study conclusions. The main purpose of this study was to examine the progression of PCO in eyes after 2 different surgical methods of cataract extraction, AquaLase and NeoSoniX. The PCO quantification was done using the subjective EPCO 2000 software and the objective OSCA system of computer PCO analysis. The OSCA is a system of objective PCO assessment, which is based on locationsensitive entropy-based texture analysis of digital images. Images with no clinical PCO produce very low scores in the analysis. The possible OSCA scores range from 0 (no PCO) to approximately 15 (practical expected maximum). Typical OSCA values for images with very little or no PCO are around 0.5. Values for patients that are deemed to warrant laser capsulotomy are typically around 4 to 5 (ref. ${ }^{16}$ ). The EPCO 2000 is a computer-assisted system of PCO morphologic assessment. It incorporates planimetric and grading assessment. The selection process and grading of areas are subjective. The EPCO 2000 scores range from 0 (no $\mathrm{PCO}$ ) to 4 (maximum) (ref. ${ }^{15}$ ). Both systems of PCO analysis measure morphologic severity of PCO (extent and density); as regards the OSCA software, it is in addition with positive weighting toward the central visual axis. We suggest that this is the reason why the correlation between EPCO 2000 and OSCA results was poor over the whole follow-up period. Changes in PCO after AquaLase and NeoSoniX phacoemulsification progressed with time in this study. Progression in EPCO 2000 results was significantly more extensive than in OSCA scores. This means that PCO extent and density progressed (according to the EPCO 2000 and OSCA outcomes), while the progression of opacities in the central part of posterior capsule was slower (according to OSCA scores and to nonsignificant increase in Nd:YAG capsulotomies). The difference in the development of PCO after the AquaLase and the NeoSoniX phacoemulsification method was not significant within a longer period of time. However, the Nd:YAG laser capsulotomy rate was nonsignificantly lower after the AquaLase method over the whole follow-up period. BCVA reached its maximum in 3 years after cataract surgery, then it decreased. Neither relation between total EPCO 2000 index nor OSCA scores and postoperative BCVA for both groups was established 1,3 , and 8 years after cataract surgery. We suggest that it was because there was a very small variability in BCVA in the study (it was 0.8 or better in most cases). PCO was assessed as minimal and its influence on BCVA was probably small, which might also explain why no relation was found between OSCA results and BCVA, as well as EPCO 2000 outcomes and BCVA.

\section{CONCLUSIONS}

A comparison of PCO after AquaLase and NeoSoniX phacoemulsification techniques was done 1,3 , and 8 years after surgery. The AquaLase method was slightly better in the majority of observed parameters (EPCO 2000 and OSCA results, Nd:YAG laser capsulotomy rate, BCVA); however, the difference was not significant in most cases. The OSCA system carries out complete PCO analysis; higher the score, the more PCO are in the central part of posterior capsule. Missing values caused by laser capsulotomies have greater impact on the OSCA outcomes than on the EPCO 2000 results. In conclusion, our study has shown that there was only minimal PCO difference between these 2 approaches, AquaLase and NeoSoniX. Both methods of cataract extraction are effective and safe. However, neither AquaLase nor NeoSoniX technique was able to prevent a natural progression of PCO.

Acknowledgement: Supported by Charles University Programme P37/07 (PRVOUK).

Author contributions: AS, JN: literature search, manuscript writing and data collection; NJ, PR: final text correction.

Conflict of interest statement: The authors state that there are no conflicts of interest regarding the publication of this article.

\section{REFERENCES}

1. Born CP, Ryan DK. Effect of intraocular lens optic design on posterior capsular opacification. J Cataract Refract Surg 1990;16(2):188-92.

2. Percival SPB, Setty SS. Analysis of the need for secondary capsulotomy during a five-year follow-up. J Cataract Refract Surg 1988;14(4):379-82.

3. Auffarth GU, Brezin A, Caporossi A, Lafuma A, Mendicute J, Berdeaux G, Smith AF; European PCO Study Group. Comparison of Nd: YAG capsulotomy rates following phacoemulsification with implantation of PMMA, silicone, or acrylic intraocular lenses in four European countries. Ophthalmic Epidemiol 2004;11(4):319-29.

4. Greenberg PB, Tseng VL, Wu WC, Liu J, Jiang L, Chen CK, Scott IU, Friedmann PD. Prevalence and predictors of ocular complications associated with cataract surgery in United States veterans. Ophthalmology 2011;118(3):507-14.

5. Dangel ME, Kirkham SM, Phipps MJ. Posterior capsule opacification in extracapsular cataract extraction and the triple procedure: a comparative study. Ophthalmic Surg 1994;25(2):82-7.

6. Prajna NV, Ellwein LB, Selvaraj S, Manjula K, Kupfer C. The Madurai Intraocular Lens Study IV: posterior capsule opacification. Am J Ophthalmol 2000;130(3):304-9.

7. Buehl W, Heinzl H, Mittlboeck M, Findl O. Statistical problems caused by missing data resulting from neodymum: YAG laser capsulotomies in long-term posterior capsule opacification studies. J Cataract Refract Surg 2008;34(2):268-73.

8. Nishi O, Nishi K, Akura J, Nagata T. Effect of round-edged acrylic intraocular lenses on preventing posterior capsule opacification. J Cataract Refract Surg 2001;27(4):608-13. 
9. Nishi O, Nishi K, Wickstrom K. Preventing lens epithelial cell migration using intraocular lenses with sharp rectangular edges. J Cataract Refract Surg 2000;26(10):1543-9.

10. Peng Q, Visessook N, Apple DJ, Pandey SK, Werner L, EscobarGomez M, Schoderbek R, Solomon KD, Guindi A. Surgical prevention of posterior capsule opacification. Part 3: Intraocular lens optic barrier effect as a second line of defense. J Cataract Refract Surg 2000;26(2):198-213.

11. Prosdocimo G, Tassinari G, Sala M, Di Biase A, Toschi PG, Gismondi M Corbanese U. Posterior capsule opacification after phacoemulsification: silicone CeeON Edge versus acrylate AcrySof intraocular lenses. J Cataract Refract Surg 2003;29:1551-5.

12. Jana Nekolová, Nad’a Jirásková, Jana Pozlerová, Pavel Rozsíval. Three-Year Follow-up of Posterior Capsule Opacification Afte AquaLase and NeoSoniX Phacoemulsification. Am J Ophthalmol 2009;148(3):390-5.e2.

13. Buratto $L$, editor. Phacoemulsification. Principles and techniques. Thorofare, New Jersey: SLACK Inc, 1998:3-21.

14. Mackool RJ, Brint SF. AquaLase: a new technology for cataract extraction. Curr Opin Ophthalmol 2004;15(1):40-3.

15. Tetz MR, Auffarth GU, Sperker M, Blum M, Völcker HE. Photographic image analysis system of posterior capsule opacification. J Cataract Refract Surg 1997;23(10):1515-20.

16. Aslam TM, Patton N, Rose ChJ. OSCA: a comprehensive open-access system of analysis of posterior capsular opacification. BMC Ophthalmol 2006;6:30.

17. Mester U, Fabian E, Gerl R, Hunold W, Hütz W, Strobel J, Hoyer H, Kohnen T. Posterior capsule opacification after implantation of CeeOn Edge 911A, PhacoFlex SI-40NB, and AcrySof MA60BM lenses J Cataract Refract Surg 2004;30(5):978-85.

18. BuehI W, Findl O, Neumayer T, Georgopoulos M, Sacu S. Short-term changes in the morphology of posterior capsule opacification. J Cataract Refract Surg 2005;31(5):962-8.

19. Hollick EJ, Spalton DJ, Ursell PG, Pande MV, Barman SA, Boyce JF Tilling K. The effect of polymethylmethacrylate, silicone, and polyacrylic intraocular lenses on posterior capsular opacification 3 years after cataract surgery. Ophthalmology 1999;106(1):49-54.
20. Mackool RJ, Brint SF. AquaLase: a new technology for cataract extraction. Curr Opin Ophthalmol 2004;15(1):40-3.

21. Hoffman RS, Fine IH, Packer M. New phacoemulsification technology. Curr Opin Ophthalmol 2005;16(1):38-43.

22. Jirásková N, Rozsíval P. Phacoemulsification parameters: series 20000 legacy versus legacy with AdvanTec software and NeoSonix handpiece. J Cataract Refract Surg 2004;30(1):144-8.

23. Jirásková N, Kadlecová N, Rozsíval P, Nekolová J, Pozlerova J, Dúbravská Z. Comparison of the effect of AquaLase and NeoSoniX phacoemulsification on the corneal endothelium. J Cataract Refract Surg 2008;34(3):377-82.

24. Lehmann RP. AquaLase versus Phaco. In: Boyd S, Dodick J, Freitas $\mathrm{LL}$, editors. New outcomes in cataract surgery. English edn. Panama City: The Highlights Coll, 2005:79-84.

25. Barsam A, Chandra A, Bunce C, Whitefield LA. Prospective randomized controlled trial to compare the effect on the macula of AquaLase liquefaction and ultrasound phacoemulsification cataract surgery. J Cataract Refract Surg 2008;34(6):991-5.

26. Davison JA. Ultrasonic power reduction during phacoemulsification using adjunctive NeoSoniX technology. J Cataract Refract Surg 2005;31(5):1015-9.

27. Ebihara Y, Kato S, Oshika T, Yoshizaki M, Sugita G. Posterior capsule opacification after cataract surgery in patients with diabetes mellitus. J Cataract Refract Surg 2006;32(7):1184-7.

28. Mian SI, Fahim K, Marcovitch A, Gada H, Musch DC, Sugar A. Nd:YAG capsulotomy rates after use of the AcrySof acrylic three-piece and one-piece intraocular lenses. Br J Ophthalmol 2005;89(11):1453-7.

29. Buehl W, Sacu S, Findl O. Association between intensity of posterior capsule opacification and contrast sensitivity. Am J Ophthalmol 2005;140(5):927-30.

30. Hancox J, Spalton D, Heatley C, Jayaram H, Yip J, Boyce J, Marshall J. Fellow-eye comparison of posterior capsule opacification rates after implantation of $1 \mathrm{CU}$ accommodating and AcrySof MA 30 monofocal intraocular lenses. J Cataract Refract Surg 2007;33(3):413-7. 